\title{
Two-Pion Production in Proton-Proton Collisions - Experimental Total Cross Sections and their Isospin Decomposition
}

\author{
T. Skorodko a , M. Bashkanov ${ }^{\mathrm{a}}$, D. Bogoslawsky ${ }^{\mathrm{b}}$, H. Calén ${ }^{\mathrm{c}}$, H. Clement ${ }^{\mathrm{a}}$, E. Doroshkevich ${ }^{\mathrm{a}}$, \\ L. Demiroers ${ }^{\mathrm{d}}$, C. Ekström ${ }^{\mathrm{c}}$, K. Fransson ${ }^{\mathrm{c}}$, L. Gustafsson ${ }^{\mathrm{e}}$, B. Höistad ${ }^{\mathrm{e}}$, G. Ivanov $^{\mathrm{b}}$, M. Jacewicz $^{\mathrm{e}}$, \\ E. Jiganov ${ }^{b}$, T. Johansson ${ }^{\mathrm{e}}$, O. Khakimova ${ }^{\mathrm{a}}$, S. Keleta ${ }^{\mathrm{e}}$, I. Koch ${ }^{\mathrm{e}}$, F. Kren ${ }^{\mathrm{a}}$, S. Kullander ${ }^{\mathrm{e}}$, A. Kupśćc \\ P. Marciniewski ${ }^{c}$, R. Meier ${ }^{\mathrm{a}}$, B. Morosov $^{\mathrm{b}}$, C. Pauly ${ }^{\mathrm{f}}$, H. Petrén. $^{\mathrm{e}}$, Y. Petukhov ${ }^{\mathrm{b}}$, A. Povtorejko ${ }^{\mathrm{b}}$, \\ R.J.M.Y. Ruber ${ }^{\text {, K. Schönning }}$, W. Scobel ${ }^{\mathrm{d}}$, B. Shwartz ${ }^{\mathrm{g}}$, J. Stepaniak ${ }^{\mathrm{h}}$, P. Thörngren-Engblom ${ }^{\mathrm{e}}$, \\ V. Tikhomirov ${ }^{\mathrm{b}}$, G.J. Wagner ${ }^{\mathrm{a}}$, M. Wolke ${ }^{\mathrm{e}}$, A. Yamamoto ${ }^{\mathrm{i}}$, J. Zabierowski ${ }^{\mathrm{h}}$, and J. Zlomanczuk ${ }^{\mathrm{e}}$ \\ a Physikalisches Institut der Universität Tübingen, D-72076 Tübingen, Germany \\ ${ }^{\mathrm{b}}$ Joint Institute for Nuclear Research, Dubna, Russia \\ ${ }^{\mathrm{c}}$ The Svedberg Laboratory, Uppsala, Sweden \\ ${ }^{\mathrm{d}}$ Hamburg University, Hamburg, Germany \\ eUppsala University, Uppsala,Sweden \\ ${ }^{\mathrm{f}}$ Forschungszentrum Jülich, Germany \\ 'Budker Institute of Nuclear Physics, Novosibirsk, Russia \\ ${ }^{\mathrm{h}}$ Soltan Institute of Nuclear Studies, Warsaw and Lodz, Poland \\ ${ }^{\mathrm{i} H i g h}$ Energy Accelerator Research Organization, Tsukuba, Japan
}

The two-pion production in pp-collisions has been investigated at CELSIUS in exclusive measurements from threshold up to $T_{p}=1.36 \mathrm{GeV}$. Total and differential cross sections have been obtained for the channels $p n \pi^{+} \pi^{0}$, $p p \pi^{+} \pi^{-}, p p \pi^{0} \pi^{0}$ and also $n n \pi^{+} \pi^{+}$. For intermediate incident energies $T_{p}>1 \mathrm{GeV}$, i.e. in the region which is beyond the Roper excitation but at the onset of $\Delta \Delta$ excitation, the total $p p \pi^{0} \pi^{0}$ cross section falls behind theoretical predictions by as much as an order of magnitude near $1.2 \mathrm{GeV}$, whereas the $n n \pi^{+} \pi^{+}$cross section is a factor of five larger than predicted. An isospin decompostion of the total cross sections exhibits a $s$-channel-like energy dependence in the region of the Roper excitation as well as a significant contribution of an isospin $3 / 2$ resonance other than the $\Delta(1232)$. As possible candidates the $\Delta(1600)$ and the $\Delta(1700)$ are discussed.

Two-pion production in nucleon-nucleon collisions is an outstanding subject, since it connects $\pi \pi$ dynamics with baryon and baryon-baryon degrees of freedom. There is increasing evidence that the puzzling ABC effect observed in doublepionic fusion reactions may possibly be traced back to an isoscalar resonance phenomenon as source for the peculiar pion pair production in the $\pi \pi$ scalar-isoscalar state $1 / 234$. By contrast the isovector $\pi \pi$ channel in double-pionic fusion be- haves regularily, i.e. shows no ABC effect and follows the expectations from conventional t-channel $\Delta \Delta$ calculations $[5$.

In view of the challenging explanation 2234 offered for the $\mathrm{ABC}$ effect it is interesting to study for comparison the behavior of $\pi \pi$ production in isoscalar, isovector and isotensor $\pi \pi$ channels in those cases, where the two actively participating nucleons do not fuse into a final nuclear bound system. From previous work it is known that the 
near-to-threshold behavior of $p p \rightarrow p p \pi^{+} \pi^{-}$and $p p \rightarrow p p \pi^{0} \pi^{0}$ channels is well understood as being dominated by excitation and decay of the Roper resonance 6778910 . At higher proton beam energies $T_{p}>1.2 \mathrm{GeV}$ theoretical calculations [6] predict the t-channel $\Delta \Delta$ excitation to take over the dominant role. These calculations are compared in Figs. 1 and 2 with the available total cross section data [11|12/13|14|15|16|17|18|19|20] including the new data from this work for the energy region from threshold up to $T_{p}=2.2 \mathrm{GeV}$. Beyond this energy the available sparse data indicate that the cross sections essentially saturate.

As already demonstrated previously [11/19] the experimentally obtained total cross sections for the various channels of the $N N \pi \pi$ system are linked by isospin relations 1121, which may be used for a model-independent isospin decomposition of the total cross sections. Such a decomposition in turn provides valuable insight into the participating reaction mechanisms, in particular into the quest, which resonances contribute.

For the $p p$ incident channel we have four possible $N N \pi \pi$ exit channels: $p p \pi^{+} \pi^{-}, p p \pi^{0} \pi^{0}$, $p n \pi^{+} \pi^{0}$ and $n n \pi^{+} \pi^{+}$. For these the following isospin relations apply:

$$
\begin{aligned}
& \sigma_{n n \pi^{+} \pi^{+}}=\frac{3}{20}\left|M_{121}\right|^{2} \\
& \sigma_{p p \pi^{0} \pi^{0}}=\frac{1}{60}\left|M_{121}-\sqrt{5} M_{101}\right|^{2} \\
& \sigma_{p p \pi^{+} \pi^{-}}=\frac{1}{120}\left|M_{121}+2 \sqrt{5} M_{101}\right|^{2}+\frac{1}{8}\left|M_{111}\right|^{2} \\
& \sigma_{p n \pi^{+} \pi^{0}}=\frac{3}{40}\left|M_{121}\right|^{2}+\frac{1}{8}\left|M_{111}\right|^{2}+\frac{1}{4}\left|M_{011}\right|^{2},
\end{aligned}
$$

where $M_{I_{N N}^{f} I_{\pi \pi} I_{N N}^{i}}$ denotes the reduced matrix element for the isospin $I_{\pi \pi}$ of the pion pair and for the isospins $I_{N N}^{f}$ and $I_{N N}^{i}$ of the nucleon pair in final and incident channels, respectively. As we see from eq. (1), the simplest situation with regard to isospin decomposition is given for the $n n \pi^{+} \pi^{+}$channel, which depends only on a single matrix element. We also note that in most cases the matrix elements enter incoherently in the total cross sections 1. Only in the $p p \pi^{0} \pi^{0}$

\footnotetext{
${ }^{1}$ Note that in differential cross sections the isospin matrix elements in general enter coherently, which complicates the isospin decomposition there. Hence we stick here to the treatment of total cross sections and leave the discussion of the differential cross sections for a forthcoming paper
}

and $p p \pi^{+} \pi^{-}$channels the matrix elements $M_{121}$ and $M_{101}$ enter coherently, i. e. lead to an interference term. Since in the latter the relative phase $\phi$ between both matrix elements enters, we may rewrite the expressions for the $p p \pi^{0} \pi^{0}$ and $p p \pi^{+} \pi^{-}$channels in the following way:

$$
\begin{aligned}
\sigma_{p p \pi^{0} \pi^{0}}= & \frac{1}{60}\left|M_{121}\right|^{2}+\frac{1}{12}\left|M_{101}\right|^{2}- \\
& -\frac{1}{\sqrt{180}}\left|M_{121}\right|\left|M_{101}\right| \cos \phi \\
\sigma_{p p \pi^{+} \pi^{-}}= & \frac{1}{120}\left|M_{121}\right|^{2}+\frac{1}{6}\left|M_{101}\right|^{2}+\frac{1}{8}\left|M_{111}\right|^{2} \\
& +\frac{1}{\sqrt{180}}\left|M_{121}\right|\left|M_{101}\right| \cos \phi
\end{aligned}
$$

Thus for each incident energy we have the five parameters $\left|M_{121}\right|,\left|M_{111}\right|,\left|M_{101}\right|,\left|M_{011}\right|$ and $\phi$ to be determined from the four experimental values for the total cross sections of the channels $p p \pi^{+} \pi^{-}, p p \pi^{0} \pi^{0}, p n \pi^{+} \pi^{0}$ and $n n \pi^{+} \pi^{+}$. Since this system is underdetermined, there is a priory no unique solution and we need additional physics input. Such a piece of input is provided by the information that $\left|M_{111}\right|$ got to be small compared to $\left|M_{101}\right|$ for three reasons: First of all, $\left|M_{111}\right|$ must vanish in the near-threshold region, since by Bose symmetry isovector $\pi \pi$ pairs must be associated with $p$-waves, which vanish at threshold 2 . Second, the Roper excitation, which is the leading process in the near-threshold region 667/8910], contributes only a tiny fraction of its strength to $\left|M_{111}\right|$ and finally, the $\Delta \Delta$ process, which is the leading process at higher energies does not contribute at all to $\left|M_{111}\right|$. From this we conclude that $\left|M_{111}\right|$ must play a minor role and the main difference between $\sigma_{p p \pi^{0} \pi^{0}}$ and $\sigma_{p p \pi^{+} \pi^{-}}$cross sections must be associated to the interference term, which then fixes $\phi$ (see eq. (2)).

This suggests the following strategy: The total cross sections of the $n n \pi^{+} \pi^{+}$channel uniquely determines $\left|M_{121}\right|$ at each incident energy. Having fixed the phase $\phi$ from inspection of $p p \pi^{+} \pi^{-}$ and $p p \pi^{0} \pi^{0}$ cross sections we can derive $\left|M_{101}\right|$

\footnotetext{
${ }^{2}$ Indeed, in the near-threshold region, which is well covered by the exclusive measurements at CELSIUS and COSY, we find to good approximation$$
\sigma_{p p \pi^{+} \pi^{-}}\left(T_{p}\right)=2 \sigma_{p p \pi^{0} \pi^{0}}\left(T_{p}-20 M e V\right)
$$

for $T_{p}<800 \mathrm{MeV}$ within experimental uncertainties, where the $20 \mathrm{MeV}$ correction of the lab beam energy $T_{p}$ accounts for the different reaction thresholds due to different $\pi^{ \pm}$and $\pi^{0}$ masses. I.e. in this region both cross sections are well explained by $M_{101}$ solely.
} 

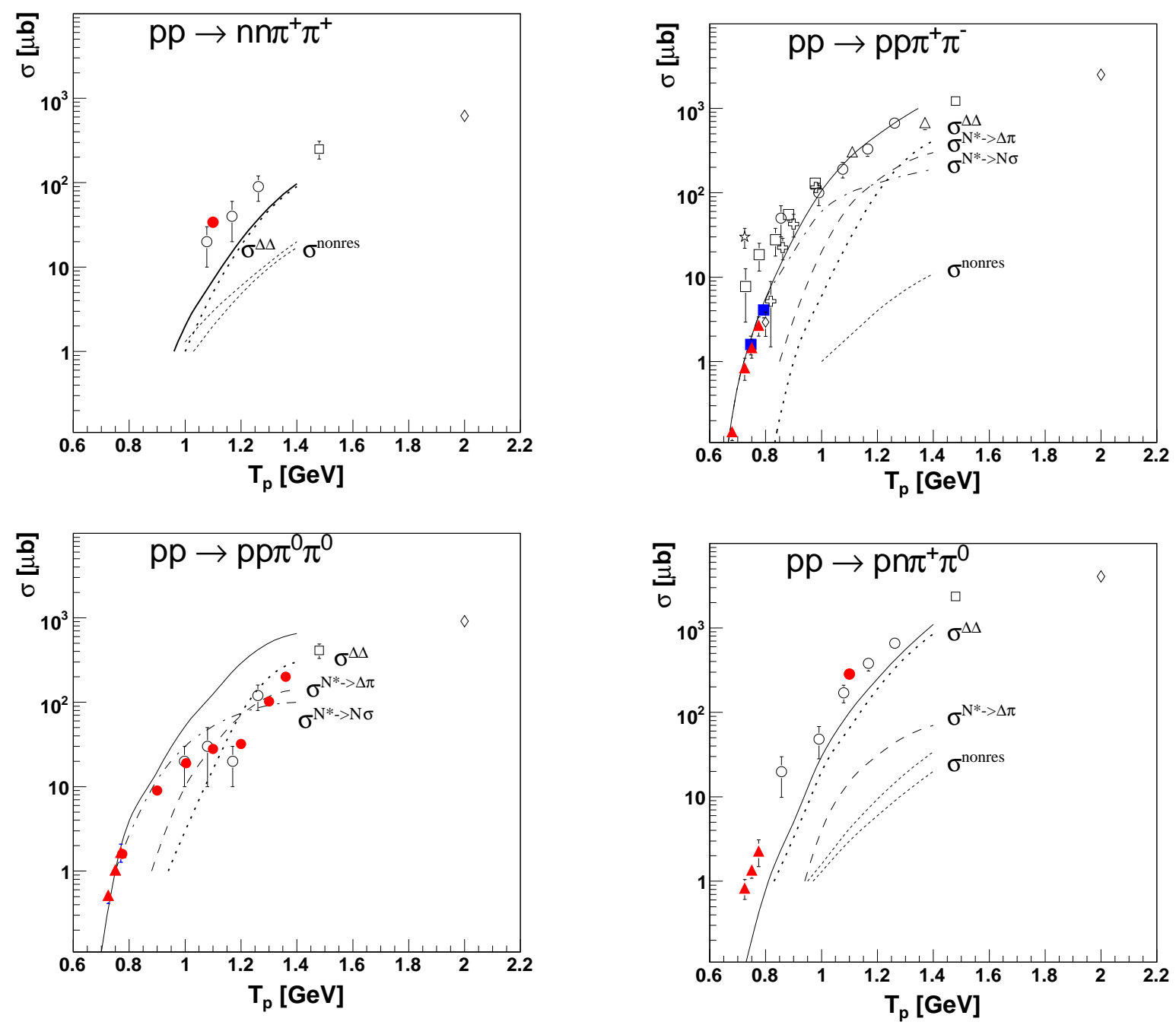

Figure 1. Energy dependence of the total cross sections for the $p p \rightarrow n n \pi^{+} \pi^{+}$(top) and $p p \rightarrow$ $p p \pi^{0} \pi^{0}$ (bottom) reactions. Thick solid dots represent the experimental results of this work. The filled triangles give PROMICE/WASA results 19. Open symbols denote previous bubblechamber measurements [15]17/18]19]. The drawn curves show the predictions of Ref. [6] for nonand semi-resonant (short dashed: contributions from diagrams (1) - (3) and (6), (7), respectively in Ref. [6]), $\Delta \Delta$ (jdotted) and Roper $\left(N^{*} \rightarrow N \sigma\right.$ : short and long dashed, $N^{*} \rightarrow \Delta \pi$ : long dashed) excitation processes. The solid lines denote the full calculations.

Figure 2. The same as Fig. 1 but for for the $p p \rightarrow p p \pi^{+} \pi^{-}(\mathbf{t o p})$ and $p p \rightarrow p n \pi^{+} \pi^{0}$ (bottom) reactions. The data are from Refs. [11, 1213 14 15 16:17/18/1920 and this work (solid dot). The open symbols denote bubble-chamber 111415 15 16 17[18] results and for $p p \rightarrow p p \pi^{+} \pi^{-}$ also single-arm magnetic spectrometer measurements from LAMPF 12/13. The filled triangles give PROMICE/WASA results [19], whereas filled squares (top figure only) show COSY-TOF measurements 20]. 
from the values of the $p p \pi^{0} \pi^{0}$ cross section. After having determined $\left|M_{111}\right|$ subsequently from the $p p \pi^{+} \pi^{-}$cross sections, we confront our results with the expectation of resonances excited in these reactions.

Since the available data base has been quite restricted strongly hampering previous isospin decompostion analyses, we have carried out a systematic program of exclusive two-pion production measurements in $p p$ collisions from threshold up to $T_{p}=1.36 \mathrm{GeV}$ using the WASA detector 22 with the hydrogen pellet target system at the CELSIUS storage ring of the Theodor Svedberg Laboratory in Uppsala. The detector has nearly full angular coverage for the detection of charged and uncharged particles. The forward detector consists of a thin window plastic scintillator hodoscope at the exit of the scattering chamber, followed by straw tracker, plastic scintillator quirl and range hodoscopes, whereas the central detector comprises in its inner part a thin-walled superconducting magnet containing a minidrift chamber for tracking and in its outer part a plastic scintillator barrel surrounded by an electromagnetic calorimeter consisting of $1012 \mathrm{CsI}(\mathrm{Na})$ crystals.

Protons and neutrons have been detected in the forward detector. The protons have been identified by the $\Delta \mathrm{E}-\mathrm{E}$ technique, neutrons by the condition of having no signals in the thin detectors window hodoscope, straw tracker and quirl. Charged pions and photons have been detected and identified in the central detector.

The absolute normalization of the data has been achieved by normalizing simultaneously measured elastic scattering and/or single pion production cross sections to known values.

Total as well as differential cross sections have been obtained for $p n \pi^{+} \pi^{0}, p p \pi^{+} \pi^{-}, p p \pi^{0} \pi^{0}$ and also $n n \pi^{+} \pi^{+}$channels. Here we concentrate on total cross sections, which are shown in Figs. 1 - 4 together with previous experimental results [7/8|11|12|13|14|15|16|17|18|19|20] as well as to the theoretical predictions [6]. For the $p p \pi^{0} \pi^{0}$ channel total cross section values of 1.6(1), 9(1), 19(2), 28(3), 32(4), 102(16), 198(25) $\mu b$ at $T_{p}=$ $0.775,0.9,1.0,1.1,1.2,1.3$ and $1.36 \mathrm{GeV}$ have been obtained. For $n n \pi^{+} \pi^{+}$and $p n \pi^{+} \pi^{0}$ chan- nels the results at $T_{p}=1.1 \mathrm{GeV}$ are $34(4)$ and 284(30) $\mu b$, respectively. These data are plotted in Figs. 1 - 4 by filled circles. Previous results from PROMICE/WASA [7/8/19 are shown by filled triangles and those from COSY-TOF [20] by filled squares. All these data stem from exclusive measurements of solid statistics. The other previous data denoted by open symbols in Figs. 1 - 4 are bubble chamber results, partly of very low statistics, or single-arm magnetic spectrometer measurements from LAMPF [12/13].

As we see from Figs. 1 and 2 our measurements are in good agreement with previous experimental results, in particular also with previous bubble chamber results [1517] for the $n n \pi^{+} \pi^{+}$and $p p \pi^{0} \pi^{0}$ channels, where we observe strong deviations from the theoretical predictions for $T_{p}>1$ $\mathrm{GeV}$.

Two surprising features emerge from these measurements for incident energies $T_{p}>1 \mathrm{GeV}$, i.e. in the region, which is beyond the Roper excitation, at the onset of the $\Delta \Delta$ excitation :

- Whereas the experimental $p p \pi^{0} \pi^{0}$ cross sections agree well with theoretical predictions [6] up to $T_{p} \approx 1 \mathrm{GeV}$, i.e. in the region of the Roper excitation, they disagree strongly at higher energies. The predicted cross section keeps rising smoothly with increasing $T_{p}$, however, the data level off for $T_{p}>1.0$ $\mathrm{GeV}$, falling behind the predictions by an order of magnitude near $1.2 \mathrm{GeV}$ and exhibiting then a sharp rise at $T_{p}>1.2$. This behavior is also in contrast to that observed in the $p p \pi^{+} \pi^{-}$channel, which is well described by the theoretical calculations.

- In sharp contrast the observed $n n \pi^{+} \pi^{+}$ cross sections are a factor of 5 larger than the theoretical predictions. This is very surprising, since under the assumption that $\Delta \Delta$ excitation is the dominant process we would expect the $p p \pi^{0} \pi^{0}$ cross section to be four times larger than the $n n \pi^{+} \pi^{+}$cross section by use of simple isospin arguments, since in this case both channels originate from the same intermediate $\Delta^{+} \Delta^{+}$system.

In order not to depend on model assump- 
tions we have carried out an isospin decomposition of the total cross sections as outlined above. In order to get the decomposition for all energies, which have been measured, we draw smooth curves through the data points and for the $n n \pi^{+} \pi^{+}$channel we also extrapolate to lower energies guided by the slope of the theoretical predictions (shaded curve in Fig. 3, where the vertical extension of the shaded area indicates the assumed uncertainty of the extracted isospin decomposed cross sections).

We use the following convention for showing the isospin decomposed results in Figs. 3 and 4: the results for the matrix elements are shown as their incoherent contribution to the cross section of interest. I.e., the result for, e.g., $\left|M_{121}\right|$ is shown in the plot for the $n n \pi^{+} \pi^{+}$channel as $\sigma_{121}^{++}$ $=\frac{3}{20}\left|M_{121}\right|^{2}$ and in the $p p \pi^{0} \pi^{0}$ channel as $\sigma_{121}^{00}=$ $\frac{1}{60}\left|M_{121}\right|^{2}$ etc., see shaded and dotted curves in Fig. 3 and 4, respectively.

In a first step we assume $\left|M_{111}\right|$ to be neglegible for reasons given above. Since $\left|M_{121}\right|$ is uniquely fixed by $\sigma_{n n \pi^{+} \pi^{+}}$, we derive then $\left|M_{101}\right|$ and the phase $\phi$ from $\sigma_{p p \pi^{0} \pi^{0}}$ and $\sigma_{p p \pi^{+} \pi^{-}}$. In the nearthreshold region, where these cross sections scale in good approximation like 1:2, i.e. like the $\left|M_{101}\right|$ contributions do, and where $\sigma_{121}^{00}$ and $\sigma_{121}^{+-}$are lower by two orders of magnitude, the $\cos \phi$ interference term does not contribute significantly and hence $\phi$ is not well determined in this region, However, for the region $T_{p}>1.0 \mathrm{GeV}$ we immediately see that the isoscalar-isotensor interference must be strongly constructive for $\sigma_{p p \pi^{+} \pi^{-}}$ and strongly destructive for $\sigma_{p p \pi^{0} \pi^{0}}$, in order to account for the experimental observation that $\sigma_{p p \pi^{+} \pi^{-}}$is larger than $\sigma_{p p \pi^{0} \pi^{0}}$ by up to an order of magnitude. Actually we only can arrive at a reasonable description of both channels in this region, if the interference is maximal, i.e. $\phi=0$. That way we also obtain $\left|M_{101}\right|$ from the $p p \pi^{0} \pi^{0}$ cross section plotted as $\sigma_{101}^{00}$ (shaded curve in Fig. $3)$.

The cross sections $\sigma_{101+121}$ calculated from the $\left|M_{101}\right|$ and $\left|M_{121}\right|$ contributions (including their interference) are given in Figs. 3 and 4 by solid $\left(\sigma_{t o t}^{00}=\sigma_{101+121}^{00}\right)$ and dot-dot-dashed $\left(\sigma_{101+121}^{+-}\right)$lines, respectively. We see that in the $p p \pi^{+} \pi^{-}$channel not only the near-threshold re- gion is well reproduced, but also the high-energy region, where $p p \pi^{0} \pi^{0}$ and $p p \pi^{+} \pi^{-}$data behave very differently. Unfortunately the $p p \pi^{+} \pi^{-}$data in this energy region show quite some scatter, which hampers severely a quantitative extraction of $\left|M_{111}\right|$. In fact, due to the propagation of the uncertainties in the data the uncertainties in $\sigma_{111}^{+-}$ get unpleasantly large at higher energies (shaded area in Fig.4). We only can state that, since $\sigma_{101+121}$ is low compared to the data by up to $30 \%$ in the region $0.9 \mathrm{GeV} \leq T_{p} \leq 1.2 \mathrm{GeV}$, the $\left|M_{111}\right|$ contribution must be of this order of magnitude there. A better fixation of $\left|M_{111}\right|$ definitely needs high-quality $\sigma_{p p \pi^{+} \pi^{-}}$data, which are not available at present.

Since continuing with the determination of $\left|M_{011}\right|$ that way would mean even larger uncertainties for this matrix element, we instead confront our results with the expectation from a resonance scenario based on the isospin decomposition results for $\left|M_{101}\right|$ and $\left|M_{121}\right|$.

In the resonance scenario we have the excitation of one or two resonances in the intermediate step of the reaction process, which then decay by pion emission into the $N N \pi \pi$ channels. In particular we have the excitation of the Roper or other higher-lying $N^{*}$ resonances schematically described by $p p \rightarrow N N^{*} \rightarrow N N \pi \pi$, the $\Delta \Delta$ excitation $(p p \rightarrow \Delta \Delta \rightarrow N N \pi \pi)$ and - as we shall see below - the excitation of the $\Delta(1600)$ $(p p \rightarrow N \Delta(1600) \rightarrow N \Delta \pi \rightarrow N N \pi \pi)$ or possibly also $\Delta(1700)$.

For each particular resonance scenario the reduced isospin matrix elements are linked by simple isospin relations obtained from isospin recoupling by use of $9 j$ symbols. For $N^{*}$ excitation we obtain for the decay branch $N^{*} \rightarrow N \sigma$ :

$$
M_{011}^{N^{*} \rightarrow N \sigma}=M_{111}^{N^{*} \rightarrow N \sigma}=M_{121}^{N^{*} \rightarrow N \sigma}=0
$$

and for the decay branch $N^{*} \rightarrow \Delta \pi$ :

$$
\begin{aligned}
& M_{111}^{N^{*} \rightarrow \Delta \pi}=+\frac{1}{2} M_{101}^{N^{*} \rightarrow \Delta \pi} \\
& M_{011}^{N^{*} \rightarrow \Delta \pi}=+\sqrt{\frac{1}{2}} M_{101}^{N^{*} \rightarrow \Delta \pi} \\
& M_{121}^{N^{*} \rightarrow \Delta \pi}=0
\end{aligned}
$$

The relation between both branches of the 

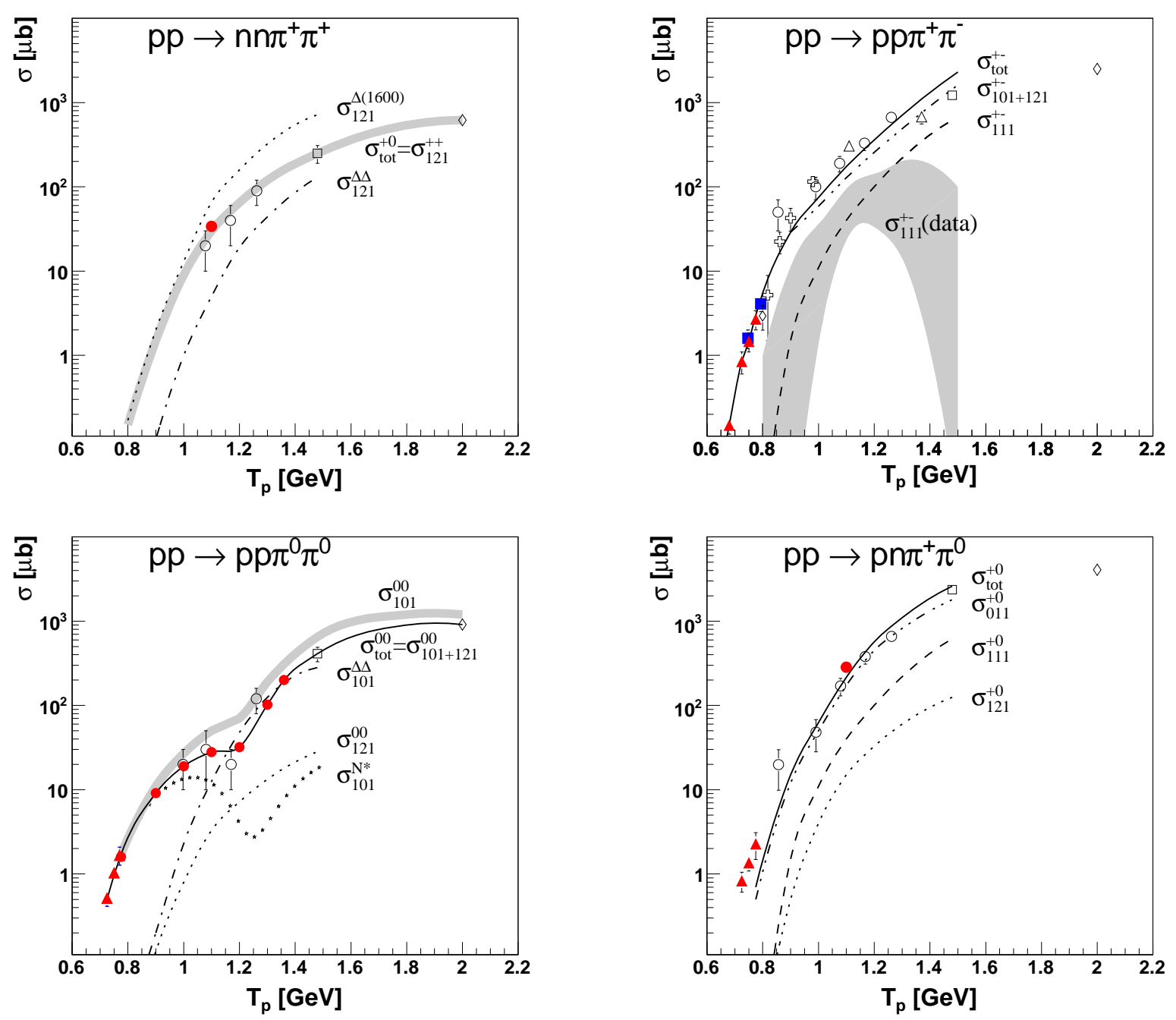

Figure 3. Isospin decompostion of the total cross sections for the $p p \rightarrow n n \pi^{+} \pi^{+}$(top) and $p p \rightarrow p p \pi^{0} \pi^{0}$ (bottom) reactions. Data are represented by open and filled symbols, see caption of Figs. 1 - 2. The drawn lines show the extracted contributions of $\sigma_{121}, \sigma_{101}$ and $\sigma_{101+121}$ as indicated in the figures. The decomposition of $\sigma_{121}$ into $\Delta(1600))$ and $\Delta \Delta$ contributions as well as of $\sigma_{101}$ into $N^{*}$ and $\Delta \Delta$ contributions are also shown. All these curves are estimated to have uncertainties of the same order as indicated by the shaded area displayed for $\sigma_{121}^{++}$(top) and $\sigma_{101}^{00}$ (bottom).

Figure 4. Same as Fig. 3, but for the $p p \rightarrow$ $p p \pi^{+} \pi^{-}$(top) and $p p \rightarrow p n \pi^{+} \pi^{0}$ (bottom) reactions. Data are represented by open and filled symbols, see caption of Figs. 1 - 2. Broken lines show the extracted contributions of $\sigma_{121}$, $\sigma_{111}, \sigma_{011}$ and $\sigma_{101+121}$ as indicated in the figures, whereas solid lines denote their sum providing the total cross sections for $p p \rightarrow p p \pi^{+} \pi^{-}$ $\left(\sigma_{t o t}^{+-}=\sigma_{101+121}^{+-}+\sigma_{111}^{+-}\right)$and $p p \rightarrow p n \pi^{+} \pi^{0}$ $\left(\sigma_{\text {tot }}^{+0}=\sigma_{011}^{+0}+\sigma_{111}^{+0}+\sigma_{121}^{+0}\right)$. The large shaded area indicates $\sigma_{111}($ data $)$ as derived by direct comparison of the $p p \rightarrow p p \pi^{+} \pi^{-}$reaction data with $\sigma_{101+121}$, whereas $\sigma_{111}$ (dashed line) is derived by use of relations (3) - (6). 
Roper process is well-known from the studies of the $p p \rightarrow p p \pi^{+} \pi^{-}$and $p p \rightarrow p p \pi^{0} \pi^{0}$ reactions in the near-threshold region $6 / 789910$. For the $\Delta \Delta$ excitation we get:

$$
\begin{aligned}
& M_{101}^{\Delta \Delta}=-\sqrt{5} M_{121}^{\Delta \Delta} \\
& M_{011}^{\Delta \Delta}=+\sqrt{\frac{15}{2}} M_{121}^{\Delta \Delta} \\
& M_{111}^{\Delta \Delta}=0
\end{aligned}
$$

and for the excitation of the $\Delta(1600)$ and its dominant decay branch $\Delta(1600) \rightarrow \Delta \pi \rightarrow N \pi \pi$ we find:

$$
\begin{aligned}
& M_{111}^{\Delta(1600)}=+\sqrt{\frac{5}{3}} M_{121}^{\Delta(1600)} \\
& M_{011}^{\Delta(1600)}=+\sqrt{\frac{10}{3}} M_{121}^{\Delta(1600)} \\
& M_{101}^{\Delta(1600)}=0 .
\end{aligned}
$$

From this we see that the two-pion decay of $N^{*}$ resonances does not contribute to $\sigma_{121}$ and hence also not to $\sigma_{p p \rightarrow n n \pi^{+} \pi^{+}}$. Therefore this cross section should be completely covered by the $\Delta \Delta$ process aside from small non-resonant contributions. As already discussed above, we face the problem that the theoretical calculations, which take into account both these processes, severely underpredict the measured data for this channel by a factor of four, which naively would mean that the $\Delta \Delta$ process ought to be larger by that factor. However, such an enlargement of this process would severely destroy any understanding of the data at high energies in the other channels as we may easily judge from inspection of Figs. 1 and 2. Hence we conclude that another resonance mechanism must be present, which strongly contributes to the $n n \pi^{+} \pi^{+}$channel and $\sigma_{121}$, respectively, but not to $\sigma_{101}$. Since only the excitation of $\mathrm{I}=3 / 2$ resonances and their successive decay into $N \pi \pi$ can fulfill these conditions, we have to look for a higher lying $\Delta$ excitation. The next higher-lying candidate is $\Delta(1600)$, which actually due to its large width of about $350 \mathrm{MeV}$ appears not unlikely to contribute already at the energies of interest. Since $\Delta(1600)$ preferably decays via $\Delta(1232)$, it contributes strongly to the isotensor cross section and also to the isovector part, but not to the isoscalar one. Similar arguments hold also for the $\Delta(1700)$, which has the disadvantage of having the pole at still higher mass, but has the advantage of a $s$-wave decay $\Delta(1700) \rightarrow \Delta \pi$.

With this hypothesis and the constraint $\phi=0$ we reconstruct the amplitude for the $\Delta(1600)$ excitation from $\sigma_{n n \pi^{+} \pi^{+}}$assuming that the theoretical prediction for the $\Delta \Delta$ process must be essentially correct both in its magnitude and in its energy dependence. The latter should be particularily reliable, since its involves the well-known $\Delta$ propagators with double $p$-wave pion emission 3 .

From eqs. (5) we see that $M_{101}^{\Delta \Delta}$ and $M_{121}^{\Delta \Delta}$ are of opposite sign, but the condition $\phi=0$ requires the complete matrix elements $M_{101}$ and $M_{121}$ to be of the same sign. Hence $\Delta \Delta$ and $\Delta(1600)$ contributions must interfere destructively in $M_{121}$. This consideration then leads to the extraction of $M_{121}^{\Delta(1600)}$, which is shown in Fig.3, top, by its incoherent contribution $\sigma_{121}^{\Delta(1600)}$ (thin dotted curve). Due to the imposed destructive interference $\sigma_{121}^{\Delta(1600)}$ must be a bit larger than $\sigma_{n n \pi^{+} \pi^{+}}$. The imposed destructive interference is actually quite reasonable, if we consider the phase behavior of the two resonances. Both $\Delta \Delta$ and $\Delta(1600)$ excitations reach their poles in the region around $T_{p} \approx 1.4 \mathrm{GeV}$. However, whereas the resonance phase for $\Delta(1600)$ runs from zero to $180^{\circ}$ as for usual resonances, the phase for the double resonance excitation $\Delta \Delta$ runs from zero to $360^{\circ}$. Hence, in the region of their dominant contributions to $N N \pi \pi$, they are out of phase supporting thus the imposed interference.

Having fixed now the issue of the unexpectedly large cross section in the $n n \pi^{+} \pi^{+}$channel, we focus next on the decomposition of $M_{101}$. Since the pole of the Roper excitation is in the region of $T_{p} \approx 0.9 \mathrm{GeV}$, Roper and $\Delta \Delta$ run now essentially with the same resonance phase in the region of interest, i.e. interfere constructively. By use of the $\Delta \Delta$ cross section from the theoretical prediction we obtain then $\sigma_{101}^{N^{*}}$ as shown in Fig. 3, bottom, by the starred curve. Whereas $\sigma_{101}^{N^{*}}$ rises up to $T_{p} \approx 0.9 \mathrm{GeV}$ as expected by the theoretical prediction for the Roper excitation, its levels

\footnotetext{
${ }^{3}$ However, we do not dare to extrapolate the behavior of the $\Delta \Delta$ cross section up to $2 \mathrm{GeV}$. Hence we stop the decompositions in partial cross sections at $1.4 \mathrm{GeV}$.
} 
off thereafter and decreases at still higher energies until it starts rising again beyond $1.3 \mathrm{GeV}$, where the excitation of the $D_{13}$ resonance might be expected. The essential observation here is that the Roper excitation exhibits an energy dependence reminiscent of a Lorentzian shape as it is adequate for a $N N^{*}$ s-channel resonance, but not for a $t$-channel associated $N^{*}$ production. However, it can not be excluded at this stage that this peculiar behavior is also possibly due to the interference of the Roper excitation with that of higher-lying $N^{*}$ states. If we compare our solution for $\sigma_{101}^{N^{*}}$ in Fig. 3 with the theoretical prediction for the $N^{*}$ excitation (dash-dotted curve Fig. 1, bottom), which shows a continously rising cross section for the Roper excitation, then we understand, why the theoretical prediction fails so badly for the $p p \pi^{0} \pi^{0}$ channel beyond $0.9 \mathrm{GeV}$.

Having understood $\sigma_{101}$ and $\sigma_{121}$ in some detail by the contributions from $N^{*}, \Delta \Delta$ and $\Delta(1600)$ excitations, we continue the discussion of $\sigma_{111}$ and extend it thereafter also to $\sigma_{011}$. Since we have fixed the various resonance contributions already by their confrontation with $\sigma_{101}$ and $\sigma_{121}$, we can calculate now $\sigma_{111}$ and $\sigma_{011}$ by use of the relations (3) - (6). The thus calculated contributions $\sigma_{111}$ and $\sigma_{011}$ are shown in Fig. 4 by the dashed and dot-dot-dot-dashed curves, respectively. Except of the highest energies $T_{p}>$ $1.3 \mathrm{GeV}$ the calculated $\sigma_{111}$ is within the region of $\sigma_{111}($ data $)$ derived directly from the data. The sum of all isospin contributions is displayed by the solid curves. We see that the data in all channels are now described quite reasonably. Even the $p n \pi^{+} \pi^{0}$ cross section in the near-threshold region is reproduced somewhat better than by the calculations of Ref. 6], since it receives now also contributions from the $\Delta(1600)$ process. Still, since all isovector terms have to vanish at threshold, even the inclusion of the $\Delta(1600)$ does not contribute sufficiently at low energies, in order to achieve a satisfying description of the data there, too. A solution of this problem may be found in the $p n$ final state interaction (FSI) not taken into account so far. In our procedure of isospin decomposition starting from the data in $n n \pi^{+} \pi^{+}$ and $p p \pi^{0} \pi^{0}$ channels we have effectively included the FSI there, since due to isospin symmetry this
FSI should be the same for $n n$ and $p p$ pairs apart from Coulomb distorsions. However, in the $p n$ system we have a much larger FSI, as borne out by the large $p n$ scattering length. Indeed, if we calculate the FSI effect on the total cross section in a Migdal-Watson ansatz 23124 based on the experimental scattering lengths and effective ranges, we obtain an enhancement of the total cross section in the near-threshold region, which in case of a $p n$ final state is roughly a factor of two larger than in case of $p p$ and $n n$ final states. Thus inclusion of the $p n$ FSI effect leads to a satisfactory understanding of the data also for the $p n \pi^{+} \pi^{0}$ channel.

In conclusion, new results for the total cross sections in $N N \pi \pi$ channels have been presented. They reveal severe shortcomings in the previous theoretical descriptions of the two-pion production reaction. The isospin decomposition based on all presently available total cross sections identify these shortcomings to be due to the effect of a higher-lying $\Delta$ resonance - most likely the $\Delta(1600)$ or possibly also the $\Delta(1700)$ - not considered in previous studies and due to the finding that the energy dependence in the region of the Roper process exhibits a peculiar $s$-channel like energy behavior.

We acknowledge valuable discussions with $\mathrm{L}$. Alvarez-Ruso, V. Anisovich, L. Dakhno, C. Hanhart, E. Oset and C. Wilkin on this issue. This work has been supported by BMBF (06TU261), Forschungszentrum Jülich and DFG (Europ. Graduiertenkolleg 683).

\section{REFERENCES}

1. M. Bashkanov et al., Phys. Lett. B637 (2006) 223

2. M. Bashkanov et al., Phys. Rev. Lett. 102 (2009) 052301; arXiv: 0806.4942 [nucl-ex]

3. M. Bashkanov et al., Proc. PANIC 08 in press; arXiv: 0906.2328 [nucl-ex]

4. H. Clement et al., Prog. Part. Nucl. Phys. 61 (2008) 276; arXiv: 0712.4125 [nucl-ex]

5. F. Kren et al., Int. J. Mod. Phys. A24 (2009) 561 and to be published

6. L. Alvarez-Ruso, E. Oset, E. Hernandez, Nucl. Phys. A633 (1998) 519 and priv. 
comm.

7. W. Brodowski et al., Phys.Rev. Lett.88 (2002) 192301

8. J. Pätzold et al., Phys. Rev. C67 (2003) 052202(R)

9. T. Skorodko et al., Prog. Part. Nucl. Phys. 61 (2008) 276

10. T. Skorodko et al., Eur. Phys. J. A35 (2008) 317

11. L. G. Dakhno et al., Sov.J. Nucl. Phys. 37 (1983) 540

12. F. H. Cverna et al., Phys. Rev. C23 (1981) 1698

13. D. R. F.Cochran et al., Phys. Rev. D6 (1972) 3085

14. C. D. Brunt et al., Phys. Rev. 187 (1969) 1856

15. F. Shimizu et al., Nucl. Phys. A386 (1982) 571

16. V. V. Sarantsev et al., Phys. Atom. Nucl. 70 (2007) 1885

17. A. M. Eisner et al., Phys. Rev. 138 (1965) B670

18. E. Pickup, D.K. Robinson, E. O. Salant, Phys. Rev. 125 (1961) 2091

19. J. Johanson et al., Nucl. Phys. A712 (2002) 75

20. S. Abd El-Bary et al., Eur. Phys. J. A37 (2008) 267

21. J.Bystricky et al., J. Physique 48 (1987) 1901

22. Chr. Bargholtz et al., Nucl. Instr. Meth A594 (2008) 339

23. A. B. Migdal, JETP 28 (1955) 1

24. K. W. Watson, Phys. Rev. 88 (1952) 1163 